\title{
Sociology of Politics
}

\author{
Lwakanwanga Muntu
}

\begin{abstract}
:
There are societal tensions all around us. They are unavoidable in human interactions. However, this does not imply that every social connection is always totally or even partially problematic. It also does not imply that every underlying problematic connection would be manifested with the same level of hatred and violence. Conflicts differ in terms of their origins, duration, manner of resolution, results, and repercussions. The emergence of specific social conflicts, battles, and struggles, rather than the function of conflict in social life, is the focus of attention. It is about disagreements between groups of people, not between individuals acting alone or inside specific groups. Finally, we are more concerned with conflicts in which coercion and violence are probable or feasible, rather than those in which coercion and violence are unlikely to occur.
\end{abstract}

Keywords: Sociology, Politics, Conflicts 


\section{Introduction}

Kuna mivutano ya jamii inayotuzunguka. Haiepukiki katika mwingiliano wa wanadamu. Walakini, hii haimaanishi kuwa kila uhusiano wa kijamii huwa shida kabisa au hata sehemu. Pia haimaanishi kuwa kila uhusiano wa msingi wa shida utaonyeshwa na kiwango sawa cha chuki na vurugu. Migogoro inatofautiana kulingana na asili yao, muda, njia ya utatuzi, matokeo, na athari. Kuibuka kwa mizozo maalum ya kijamii, vita, na mapambano, badala ya kazi ya mizozo katika maisha ya kijamii, ndio mwelekeo wa umakini. Inahusu kutokubaliana kati ya vikundi vya watu, sio kati ya watu wanaofanya peke yao au ndani ya vikundi maalum. Mwishowe, tunajali zaidi na mizozo ambayo kulazimisha na vurugu kunawezekana au kutekelezeka, badala ya zile ambazo kulazimishwa na vurugu haziwezi kutokea.

\section{Literature Review}

Yote juu yetu ni migogoro ya kijamii. Wao ni asili katika uhusiano wa kibinadamu. Lakini hii haimaanishi kwamba kila uhusiano wa kijamii unapingana kabisa au hata sehemu wakati wote. Wala haimaanishi kuwa kila uhusiano wa kimsingi unaokinzana utaonyeshwa kwa kiwango sawa na aina ya uhasama au vurugu. Migogoro inatofautiana katika misingi yao, muda wao, hali yao ya makazi, matokeo yao, na matokeo yake. Kitabu hiki ni juu ya tofauti kama hizo. Lengo la wasiwasi ni juu ya ukuzaji wa mizozo maalum ya kijamii, ya mapigano na mapambano, badala ya jukumu la mizozo katika maisha ya kijamii. Inahusu ugomvi kati ya vikundi vya watu, na sio ndani ya vikundi maalum au kati ya watu wanaofanya peke yao. Mwishowe, tunajali sana mapambano ambayo kulazimika na vurugu kunawezekana au inawezekana, kuliko zile ambazo zimedhibitiwa sana kwamba kulazimishwa na vurugu hazifanyiki. 
Kuna maoni mengi ya mshikamano wa nadharia na uhalali wa kihistoria wa nadharia ya MarxistLeninist ya ubeberu; vidokezo vichache vitatosha hapa. Kwanza, hata ikiwa mtu angekubali uhusiano kati ya ubepari na ubeberu, uhusiano wa kinadharia kati ya ubeberu na vita, haswa vita vya kati, haujawahi kuonyeshwa kwa kusadikisha. Inawezekana pia kuwa upanuzi wa kibeberu, haswa katika enzi ya mpaka wazi wa kikoloni, hupunguza uwezekano wa vita kuu kwa kugeuza ushindani mkubwa wa nguvu kutoka msingi wa mfumo kwenda pembezoni, ambapo masilahi yao muhimu hayana uwezekano wa kugongana na ambapo suluhisho za maelewano zinawezekana zaidi. Kautsky alipendekeza kuwa ushindani wa kibeberu utasababisha "ubeberu mkubwa," ushirikiano kati ya majimbo ya kibepari kwa unyonyaji wa pamoja wa pembezoni. Pili, kwa kiwango cha kijeshi, ikiwa tunachukulia uhusiano madhubuti kati ya mifumo ya kisiasa ya kidemokrasia ya huria na mifumo ya uchumi wa kibepari, nadharia ya Marxist-Leninist inafanya utabiri mbili ambao unapingana moja kwa moja na uhusiano wa kijeshi kati ya demokrasia huria na vita. Vita vilivyotabiriwa kati ya majimbo ya kibepari huria hayakuwa ya kawaida, na majimbo ya kibepari hayajawahi kukabiliwa na vita au uwezekano mkubwa wa kuanzisha vita kuliko majimbo mengine katika mfumo wa kimataifa..

Kwa upande mwingine, waangalizi wengi wanasema kuwa mizozo hutokana na kutokubaliana kwa masilahi na kwamba vyama kwa ujumla vinajua kutokulingana huko (Madison, 1937). Walakini, hata watu wanaoamini kuwa uhusiano wa kimsingi wa mizozo unasababisha ufahamu wanaweza kusema kwamba watu wakati mwingine wanaamini kwa uwongo kuwa hakuna mzozo. Katika visa kama hivyo wanaweza kusema juu ya "ufahamu wa uwongo" au kushtaki kwamba watu wanadanganywa na kudanganywa. Watu kama hao wanaweza kuendelea kusema kwamba uongozi na shirika ni muhimu ili kuwafanya washiriki wanaoweza kujua uhusiano wao unaopingana. Mafanikio ya viongozi hao katika kuhamasisha washirika na kuleta mgogoro kwa 
matokeo yanayotarajiwa, hata hivyo, bado inategemea usahihi wa viongozi katika kutafsiri hali ya malengo.

Watu katika nchi zote za kidemokrasia na zisizo za kidemokrasia mara nyingi huwa na shauku mwanzoni mwa vita, ingawa msaada huu unaweza kupungua haraka ikiwa vita vitaendelea na kugharimu. Katika siasa za Amerika uungwaji mkono maarufu kwa rais unakua kila mara baada ya matumizi ya nguvu, bila kujali hekima au mafanikio ya hatua hiyo ya kijeshi. Mfano huu umeelezewa na tabia ya umma ya kuzunguka bendera, rais, na chama, na mwishowe na hali ya utaifa wa kisasa.

Inaweza kuonekana kuwa ya busara kudhani kuwa hisia na tabia hutofautiana pamoja kwa nguvu. Mtu anaweza kutarajia kwamba ikiwa chama kimoja kinataka kumdhuru mpinzani wake, itakuwa; kwamba kadri hamu ya kihemko ya kuumiza mtu mwingine inavyoongezeka, ndivyo matumizi yake ya kulazimisha katika kuonyesha hamu hiyo. Kinyume chake, vurugu kubwa kawaida hazijatumika bila hasira kubwa. Tafakari kidogo, hata hivyo, inapaswa kufunua upungufu wa mawazo kama hayo. Kuna nyakati ambapo mmoja au wote wanaohusika kwenye mzozo wanahisi uhasama mkali lakini hawajaribu kumdhuru mwenzake. Wanaweza kuzuiliwa na hofu ya kulazimishwa au kwa imani kwamba kulazimishwa hakutakuwa na ufanisi katika kupata kile wanachotaka kutoka kwa mpinzani.

Kwa upande mwingine, katika hali zingine jeraha kubwa linaweza kusababishwa kwa mpinzani aliye na uhasama kidogo au hana uhasama wowote unaofuatana na vurugu. Hii inawezekana wakati vikundi vikubwa vinahusika katika mzozo; vurugu hufanyika kupitia teknolojia ngumu na kuna mgawanyiko mkubwa wa kazi katika kuendesha mapambano na matumizi ya vurugu. Mazingira kama haya yanaonekana wazi katika vita vya kimataifa ambapo mabomu ya juu huajiriwa. Hata kati ya askari wa vita vya watoto wachanga, hata hivyo, "chuki ya adui, ya 
kibinafsi na isiyo ya kibinadamu, haikuwa jambo kuu katika motisha ya vita," waandishi wa utafiti wa askari wa Amerika katika Vita vya Kidunia vya pili walihitimisha (Stouffer, et al., 1949, p. 166). Mauaji yasiyofaa yanaweza kuonekana kuwa ya kuchukiza sana, lakini chuki kali inaweza kuwa sababu ya vurugu zilizofanywa kibinafsi ambazo tunaziita ukatili.

Kinadharia, nadharia ya Azazeli inategemea nadharia ya kikundi / nje ya kikundi katika sosholojia. Simmel, katika matibabu ya kimfumo ya kwanza ya mada hiyo, alisema kuwa mzozo na kikundi cha nje huongeza mshikamano na ujumuishaji wa kisiasa wa kikundi hicho, na jumla kwa uhusiano wa kimataifa: "vita na nje wakati mwingine ndio nafasi ya mwisho kwa serikali iliyojaa uhasama wa ndani kushinda haya maelewano, au vinginevyo kuvunja dhahiri. " Coser alibadilisha maoni mengi ya Simmel. Anasema kuwa mshikamano wa kikundi hicho utaongezwa tu ikiwa tayari kuna kiwango kidogo cha mshikamano wa ndani na ikiwa tu inagunduliwa kuwa tishio la nje linahatarisha kikundi kwa ujumla na sio sehemu yake tu. Vinginevyo, mzozo wa nje utasababisha mzozo wa ndani na utengano badala ya mshikamano. Coser ni mamlaka iliyotajwa sana juu ya nadharia ya kikundi / nje ya kikundi, lakini sifa hii muhimu haitambuliki kila wakati.

Kwa kifupi, usafi wa mzozo hutegemea seti nzima ya uhusiano kati ya wahusika katika mzozo. Inategemea pia kiwango ambacho suala linalobishaniwa linaweza kugawanywa kuwa maswala madogo. Sifa hizi za mapambano kwa sehemu hutegemea njia ambayo wahusika kwenye mzozo wanauona - ni kwa kiwango gani imetengwa na masilahi ya kawaida au imeingizwa katika masilahi anuwai ya kawaida. Maoni haya yanaweza kubadilika wakati wa mapambano. Usafi wa mzozo, kwa upande wake, huathiri ukali wake na chaguo la njia za kufikia makubaliano ya pamoja.

Maelezo ya kimsingi ya kukosekana kwa ushahidi wa uhusiano kama huo ni kukosekana kwa mfumo wa nadharia uliokua vizuri unaoongoza masomo ya kijeshi. Sababu moja ya umaskini huu wa kinadharia ni mipaka nyembamba sana ya nidhamu na kutofaulu kwa wanasayansi wa kisiasa 
kuthamini vyanzo vyenye utajiri wa ufahamu wa nadharia katika nyanja zingine. Ingawa wanasayansi wa kisiasa mara nyingi wanakubali utumiaji unaofaa wa fasihi za kihistoria za kupima ujasusi wao wa nadharia, wanadharau matumizi yake kama chanzo cha mapendekezo ya nadharia. Fasihi zinazohusiana na nadharia ya mbuzi ni mfano bora wa hoja ya Bueno de Mesquita kwamba "mara nyingi hatulete ukali sana kwa nadharia yetu kama tunavyofanya kwa uchambuzi wetu wa data," lakini pia inaonyesha vyanzo vingi vya ufahamu muhimu wa nadharia ambayo inaweza kusaidia katika kazi ya maendeleo ya kinadharia.

Yote hii inapaswa kuonyesha ni kwanini ni ngumu kwa washiriki katika mzozo kutathmini nguvu zao. Kwa hivyo, jaribio la moja kwa moja au makabiliano mara nyingi ni muhimu kwa wahusika kutathmini nguvu zao na jinsi jambo lililo katika ubishani ni muhimu kwa kila mmoja wao. Tukigundua sehemu hizi za madaraka inapaswa pia kuelezea jinsi inavyowezekana kwa chama kimoja chenye nguvu ndogo kuhimili au hata kushinda nguvu kubwa zaidi. Hii inaweza kuonekana katika maswala ya kimataifa haswa katika kuvunja vifungo vya wakoloni. Kwa watu katika koloni, uhuru wao wa kitaifa unaweza kuonekana kuwa muhimu sana kwamba wako tayari kupata hasara kubwa wakati nguvu ya kikoloni haiko tayari kuvumilia usumbufu mwingi kwa lengo ambalo sio msingi wake.

\section{Conclusion}

Kwa kuchunguza baadhi ya fasihi anuwai ya nadharia juu ya siasa za nyumbani na mizozo, utafiti huu unachukua hatua ya kwanza kuelekea kuziba mgawanyiko huu. Inaangalia uhusiano kati ya sifa za kitaifa na tabia ya vita, uwezekano wa serikali za kidemokrasia na zisizo za kidemokrasia kwenda vitani, maoni ya Marxist na huria juu ya jukumu la muundo wa uchumi, athari ya utaifa na maoni ya umma, na nadharia ya mbuzi. Lakini kwanza, insha hii inachunguza jinsi wanasayansi wa kisiasa na wanahistoria wanavyoshughulikia asili ya ndani ya mizozo. 
